\title{
PYRAMID TYPE PLATE BENDING MACHINE
}

\author{
Vaibhav Suresh Deore ${ }^{1}$, Gauri Rajendra Bauskar², Karan Sanjay Bhadane ${ }^{3}$, \\ Siddhesh Mukesh Gaikwad ${ }^{4}$ \\ ${ }^{1}$ Development Engineer at TALCO India Nasik \\ ${ }^{2}$ Student of SYMBIOSIS Institute of Technology \\ ${ }^{3}$ Student of N.D.M.V.P'S College of Engineering \\ ${ }^{4}$ Student of N.D.M.V.P'S College of Engineering
}

\begin{abstract}
Bending machine is a common machine that is used to bend a metal. It is most frequently used in various industrial processes such as bending a sheet to make cylinders or to make various shape such as "V shape". There are many kind of bending machine that can be found in the market such as press brake bending machine. Roll bending machine, and folding bending machine. In this paper design a bending machine specially for the bending a sheet metal is given. There is no proper small scale bending machine for bending a sheet metal having thickness up to $2 \mathrm{~mm}$ is manufactured. In this paper all the necessary criterion of small scale bending machine to be considered such as roller bending type to be used, the design of the bending machine, the method of assembly the machine parts and the consideration of material need to be bend. A idea to design a bending machine is followed in the paper by considering design parameters.
\end{abstract}

Keywords:-Bending machine, working principle, design, materials, material selection, and Stress analysis $* * *$

\section{INTRODUCTION}

A Plate Rolling Machine is a machine that will roll different kind of metal sheet into a round or conical shape. It can be also called "Roll bending machine" "plate bending Machine" or "rolling machine"

They are different kinds of Technology to roll the metal plate: Roller machine: Anatomy; a Top-Roll, the PinchingRoll, and two Side-Rolls. The flat metal plate is placed in the machine on either side and "pre-bent" on the same side. The Side-Rolls do the work of bending. The Pinching Roll holds the plate. Roll Machines (Variable Pitch aka Variable Geometry): Anatomy; One Pressing Top-Roll, Two Pressing Side-Rolls. 3 Roll Variable Pitch aka Variable Geometry works by having all three rolls being able to move and tilt. The Top-Roll moves on the vertical plane. The Side-Rolls move on the horizontal plane.

When rolling, the Top-Roll presses the metal plate between the two Side-Rolls. The advantage of having the Variable 3 Roll is the ability to roll many thicknesses and diameters of cylinders. For example; The Side-Rolls are what produce the mechanical advantage. With the Side-Rolls all the way open, then you have the maximum mechanical advantage. With the Side-Rolls all the way in, you have the least mechanical advantage So, your machine has a capability of rolling $2 \mathrm{~mm}$ thick material with the maximum mechanical advantage, but your job is only $1 / 2 \mathrm{~mm}$ thick. Simply reduce the mechanical advantage and you have a machine that can roll from $1 / 2 \sim 2 \mathrm{~mm}$ thick.

\section{LITERATURE SURVEY}

\subsection{Overview}

A pyramid type plate bending machine is widely use in automation industry. Unfortunately, there is no proper bending machine to bend a sheet metal of less thickness. Moreover, the existing bending machine is big in size, consume a lot of space and it is use for big scale production. In this project, the literature review will discuss in detail in designing a bending machine, component features considered in designing a bending machine, etc.

Plate bending is a process by which a metal can be bend by plastically deforming the material and its shape. Material suppose to be stressed beyond the yield strength but below the ultimate tensile strength. Roll forming, [also known as roll bending], is a process for forming plates, sheets, bars, beams, angles or pipes into various shapes by passing the work piece between the properly spaced rolls. Sheet and plate are the mill products often formed by the three roll bending machine.

The elastic part of the deformation is recovered up on removal of the load. This phenomenon is known as "spring back" or elastic recovery. Metal forming processes are classified into two categories: hot working and cold working process. Plate bending is a cold working process. Cold working process is the plastic deformation of metals below the recrystallization temperature. This process is mostly done in room temperature.

Plate bending machines can be classified based on the number and arrangement of the rollers, also on the method 
of pinching the work piece. Types of three roll pyramid type plate bending machines are:

1. Single (initial) pinch 3-roll plate bending machine

2. Double pinch 3-roll plate bending machine

3. 4 roll plat bending machines.

\subsection{The Main Working Components of the Pyramid}

\section{Type Plate Bending Machine}

1. These rolls are supported on either side by the left and right stands.

2. The two bottom rollers receive power where as the top roll doesn't, it rotate freely.

3. To deform plates into cylinders or cones, the top roll must be subjected by a down ward load; this is achieved by a screw pressure through a power screw, which is located at the top of the two stands

4. The top roll have more load than the bottom rolls; it is therefore, made of a large diameter. And it is supported on a hinge (or drop end) on the right side; the hinge is also supported on the housing block and carries the bearing to support the shaft and moves together with roll and the block.

\section{WORKING PRINCIPLE}

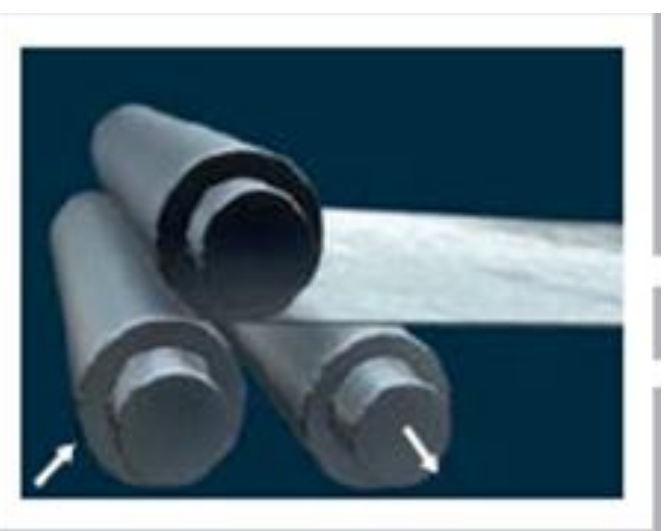

Fig 1: Insert the plate by adjusting the upper roller and by adjusting the upper roller provide the pre stress on plate

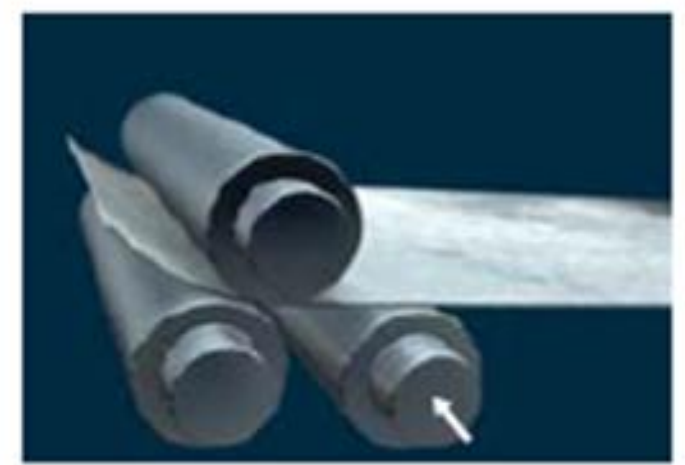

Fig 2: The plate bends towards the upper roller and after one complete cycle measure the radius

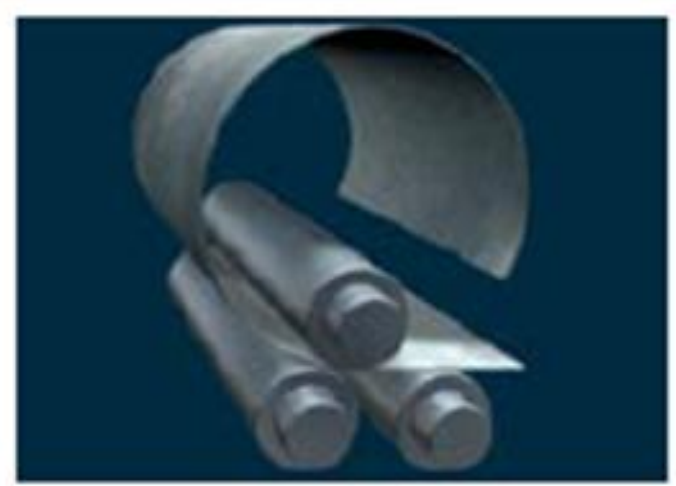

Fig 3: Roll the whole sheet as per required diameter

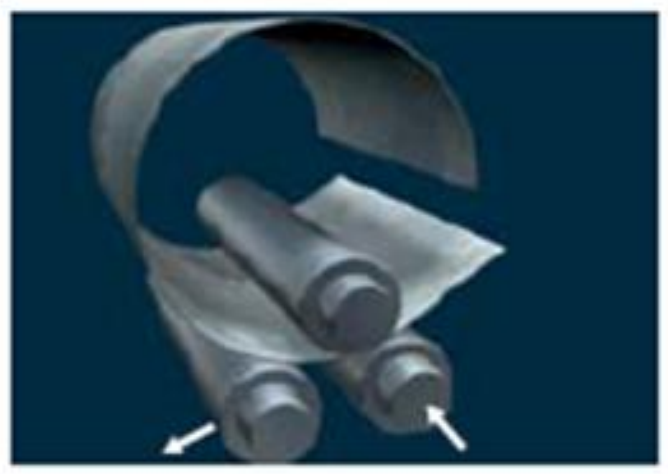

Fig 4: Adjust the rolls for the final outcome and make necessary clamping force

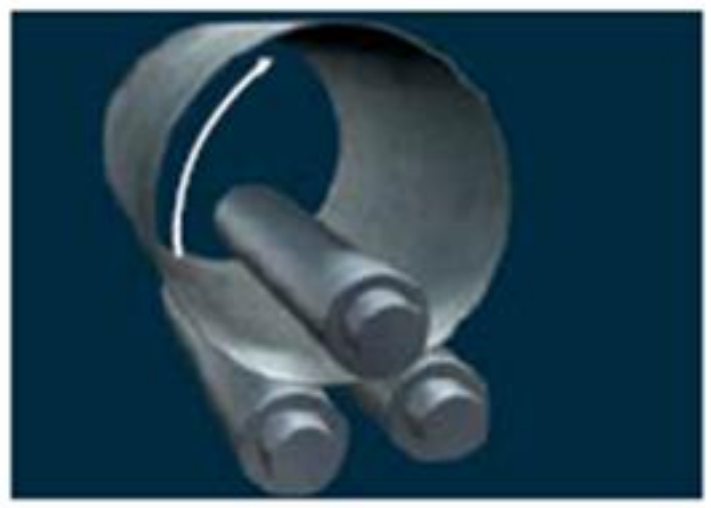

Fig 5: After getting required diameter lower the clamp force

4. MATERIAL SELECTION

AND

\section{MANUFACTURING}

\subsection{Material Selection}

\subsubsection{Criteria for material selection:}

Selection of material is one of the most important factors which must be considered seriously for successful fabrication of any component of pyramid plate bending machine 
The material selection must have following desirable characteristics:-

1. It should possess modulate strength, toughness in working condition.

2. It should sustain vibration caused by rotating parts.

3. It should have attractive outlook.

4. It should be least in weight.

5. Material should retain its strength under loading.

6. It should be fulfilling from economical point of view.

\subsubsection{Material Selected}

As mentioned earlier the selection of material plays an important role in the development of the model. The efficiency and the performance of a plate rolling machine depend upon the material used for manufacture of its component. Thus we have selected materials for the respective components as below:-

Table 1: Materials Selected

\begin{tabular}{|l|l|l|}
\hline Component & Material & Properties \\
\hline $\begin{array}{l}\text { Stand and base } \\
\text { plate }\end{array}$ & Mild steel & $\begin{array}{l}\text { Easily Available } \\
\text { Easily welded } \\
\text { Durable }\end{array}$ \\
\hline $\begin{array}{l}\text { Side blocks for } \\
\text { bottom rolls }\end{array}$ & EN 8 & $\begin{array}{l}\text { good tensile strength } \\
\text { resistant to wear } \\
\text { easily machinable }\end{array}$ \\
\hline $\begin{array}{l}\text { Bottom and Top } \\
\text { rollers }\end{array}$ & EN 8 & $\begin{array}{l}\text { good tensile strength } \\
\text { resistant to wear } \\
\text { easily machinable }\end{array}$ \\
\hline Bush & Plain Brass & $\begin{array}{l}\text { When it comes in } \\
\text { contact with steel } \\
\text { machine shafts, it will } \\
\text { not bind to steel } \\
\text { because it is } \\
\text { dissimilar metal }\end{array}$ \\
\hline Sliding blocks & EN 8 & $\begin{array}{l}\text { good tensile strength } \\
\text { resistant to wear } \\
\text { easily machinable }\end{array}$ \\
\hline Gears & EN36 & $\begin{array}{l}\text { Hard, tough, resistant } \\
\text { to wear }\end{array}$ \\
\hline EN9 & $\begin{array}{l}\text { good tensile strength } \\
\text { resistant to wear } \\
\text { easily machinable }\end{array}$ \\
\hline
\end{tabular}

\subsection{Manufacturing Process}

\subsubsection{Bottom Rollers}

1. Cutting: Raw material is cut down into required length by using hacksaw machine.

2. Turning: Diameter of workpiece is reduced to the required diameter which is $50 \mathrm{~mm}$ on lathe machine.

3. Grinding: For finishing purpose grinding is done.

\subsubsection{Sliding Block for Top Roller}

1. Cutting: Raw material of EN8 is cut into the required dimensions by using hacksaw machine.
2. Milling: The cut blocks are then accurately shaped into the required dimensions by using milling machine.

3. Drilling \& Boring: To form the hole of $35 \mathrm{~mm}$ in the block we use drilling $\&$ then boring operation. These holes are used to mount the rollers in their proper position.

4. Press fitting: This operation is used to press fit the P.B. Bush ( plain Brass ) of dia $35 \mathrm{~mm}$ into the holes which are made in the block. This is done by hammer.

5. Boring: By using DRO (Digital Read out )milling machine a hole of dia $25 \mathrm{~mm}$ is made in press fitted P.B. bush.

6. Tapping: A 2/4 drilling tapping tool is operated by hand to thread two holes at the top of block. Holes are made up of $4 \mathrm{~mm}$ dia.

7. Slotting: A hole of 5 X $5 \mathrm{~mm}$ through the height of block with the help of milling machine.

\subsubsection{Top Roller}

1. Cutting: Raw material is cut into required dimension with the help of hacksaw machine.

2. Turning: In order to reduce the diameter of the work piece to the required diameter which is $57 \mathrm{~mm}$, turning operation is performed on lathe machine.

3. Grinding: For finishing purpose grinding is done.

\subsubsection{Spur Gears:}

I) Gear cutting Process

1. Hobbing

2. Shaping

3. Planing

4. Milling

II) Gear Finishing Process

1. Grinding

2. Shaving

III) Gear Forming Process

1. Extrusion

2. Powder Metallurgy

3. Stamping

4. Casting

\subsubsection{Square Key}

1. Cutting: Raw material is cut into required dimension with the help of hacksaw machine.

2. Milling: The cut blocks are then accurately shaped into the required dimensions by using milling machine.

\subsubsection{Sprocket}

They are basically used to transmit rotary motion of the shaft to another. In this plate rolling machine, two sprockets are provided, one sprocket is fitted on the motor shaft with the help of sunk key and grub screw and the other sprocket is fitted on the bottom roller shaft with the help of key and screw.Standard sprockets with 25 no of teeth have been selected 


\section{DESIGN}

\subsection{Bottom Rollers:}

Material: EN8 (40Mn2Si2)

Dimensions: Length $=250 \mathrm{MM}$

$$
\text { Diameter }=50 \mathrm{~mm}
$$

\section{Design procedures:}

Permissible tensile stress: $90 \mathrm{~N} / \mathrm{mm}^{2}$

Permissible shear stress: $65 \mathrm{~N} / \mathrm{mm}^{2}$

Permissible compressive stress: $110 \mathrm{~N} / \mathrm{mm}^{2}$

$$
\begin{aligned}
& \text { Power }=1 \mathrm{HP}=746 \mathrm{~W} \\
& \mathrm{~N}=80 \mathrm{rpm} \\
& \mathrm{P}=2 \pi \mathrm{NT} / 60 \\
& 746=2 \pi \times 80 \times \mathrm{T} / 60 \\
& \mathrm{~T}=89.047 \times 10^{3} \mathrm{~N}-\mathrm{mm}
\end{aligned}
$$

In actual practice rollers are subjected to shock and fatigue loading

Hence design is accounted by using the factors known as combined shock and fatigue factors.

Hence $\mathrm{k}_{\mathrm{b}}=1.5$ and $\mathrm{k}_{\mathrm{t}}=1$ .as load is gradual

Bending moment

$\mathrm{M}=\mathrm{F} \times \mathrm{L} / 4$

$=400 \times 250 / 4$

$=25 \times 10^{3} \mathrm{~N}-\mathrm{mm}$

Equivalent Twisting Moment,

$$
\begin{aligned}
\mathrm{T}_{\mathrm{e}} & =\sqrt{(\mathrm{Kb} \times \mathrm{M})^{2}+(\mathrm{Kt} \times \mathrm{T})^{2}} \\
& =\sqrt{(1.5 \times 25 \times 103)^{2}+(1 \times 89.047 \times 103)^{2}} \\
& =96.62 \times 10^{3} \mathrm{~N}-\mathrm{mm}
\end{aligned}
$$

According to max. Shear stress theory,

$$
\begin{aligned}
\mathrm{T}_{\max } & =16 \mathrm{~T}_{\mathrm{e}} / \pi \mathrm{d}^{3} \\
& =\left(16 \times 96.62 \times 10^{3}\right) /\left(\pi \times 50^{3}\right) \\
& =3.93 \mathrm{~N}-\mathrm{mm}^{2}
\end{aligned}
$$

Since the value of $\mathrm{G}_{\max }$ is less than permissible shear stress i.e $\quad \mathrm{G}_{\max }<\mathrm{T}_{\mathrm{per}}$

Hence, the design is safe

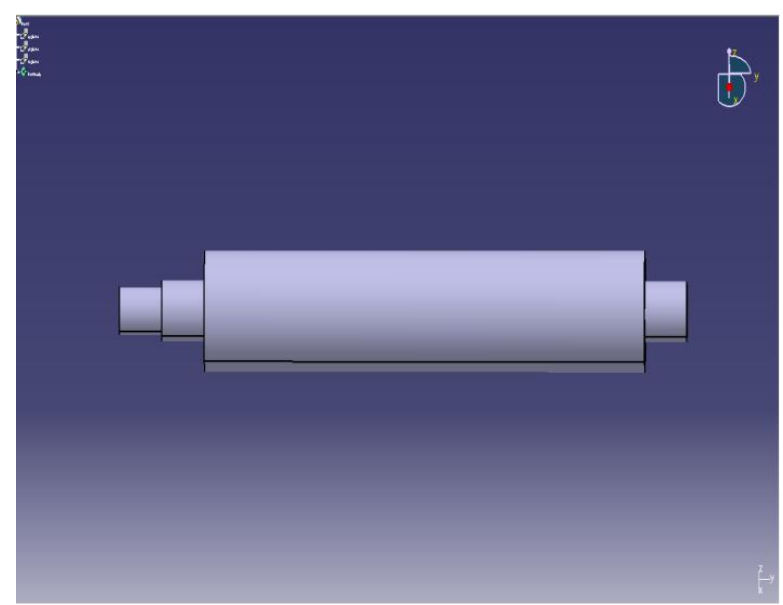

Fig 6

\subsection{Sliding Block for Top Roller:}

Material: $\mathrm{EN} 8\left(\mathrm{M}_{2} \mathrm{Si}_{12}\right)$

Dimensions: Length $=60 \mathrm{~mm}$

Height $=60 \mathrm{~mm}$

Width $=20 \mathrm{~mm}$

\section{Design Procedure:}

Permissible compressive stress: $110 \mathrm{~N} /$ square mm.

Total Force $=$ Weight of block x $9.81+$ Force transmitted by man.

$\begin{aligned} \text { Total Force } & =1 \times 9.81+400 \\ & =409.81\end{aligned}$

$$
=410 \mathrm{~N}
$$

Area of block $=$ Area of block $-\quad$ A Area of hole $+2($ Area of slot) ]

$$
\begin{gathered}
=60 \times 60-\left[\pi / 4 \times 35^{2}+2(5 \times 60)\right] \\
=2037.88=2038 \mathrm{~mm}^{2}
\end{gathered}
$$

Now,

$$
\begin{aligned}
\sigma & =\mathrm{F} / \mathrm{A} \\
& =410 / 2038 \\
& =0.20 \mathrm{~N} / \mathrm{mm}^{2}
\end{aligned}
$$

As $\sigma_{\mathrm{c}}>\sigma_{\text {per, }}$ design is safe.

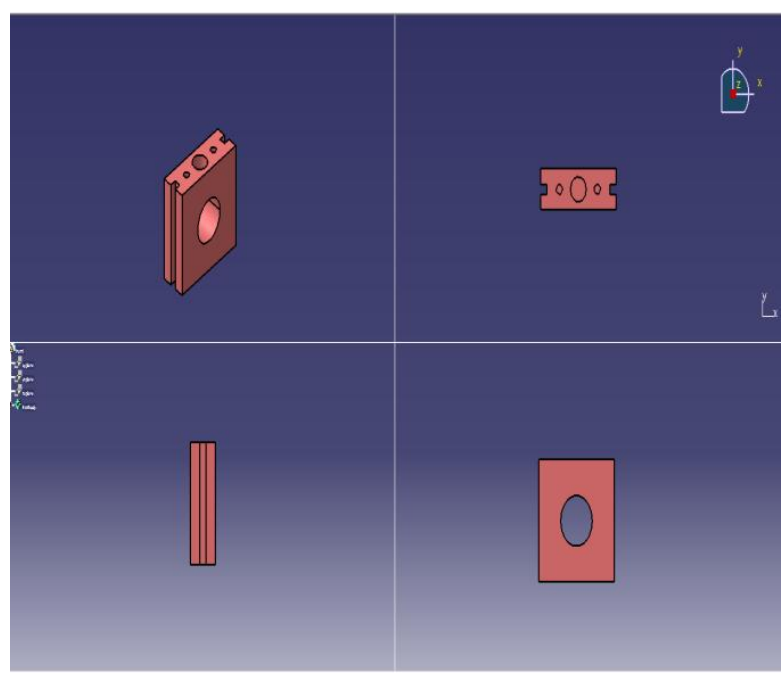

Fig 7

\subsection{Top Roller}

Material: EN8 (40Mn2Si12)

Dimensions: Length: $250 \mathrm{~mm}$

Diameter: $57 \mathrm{~mm}$

\section{Design procedure:}

Permissible tensile stress: $90 \mathrm{~N} / \mathrm{mm}^{2}$

Permissible shear stress: $65 \mathrm{~N} / \mathrm{mm}^{2}$

Permissible compressive stress: $110 \mathrm{~N} / \mathrm{mm}^{2}$

Now,

$$
\begin{aligned}
& \text { Power }=1 \mathrm{HP}=746 \mathrm{~W} \\
& \mathrm{~N}=80 \mathrm{rpm} \\
& \mathrm{P}=(2 \pi \times \mathrm{N} \times \mathrm{T}) / 60 \\
& 746=(2 \pi \times 80 \times \mathrm{T}) / 60 \\
& \mathrm{~T}=89.047 \times 10^{3} \mathrm{~N}-\mathrm{mm}
\end{aligned}
$$


Design is accounted using the factors known as combined shock and fatigue factors.

Hence,

$$
\begin{aligned}
& \mathrm{K}_{\mathrm{b}}=1.5 \\
& \mathrm{~K}_{\mathrm{t}}=1 \quad \ldots \ldots \ldots \text { (As loading is gradual) }
\end{aligned}
$$

According to maximum shear stress theory,

$$
T_{\max }=\left(16 \times \mathrm{T}_{\mathrm{e}}\right) /\left(\pi \times \mathrm{d}^{3}\right)
$$

Bending Moment $=(\mathrm{F} x \mathrm{l}) / 4$

$$
\begin{aligned}
& =(400 \times 250) / 4 \\
& =25 \times 10^{3} \mathrm{~N}-\mathrm{mm}
\end{aligned}
$$

Equivalent Twisting Moment,

$$
\begin{aligned}
& \mathrm{T}_{\mathrm{e}}=\sqrt[2]{(\mathrm{Kb} \mathrm{M})^{2}+(\mathrm{Kt} \mathrm{T})^{2}} \\
& =96.62 \times 10^{3} \mathrm{~N}-\mathrm{mm}
\end{aligned}
$$

Now,

$$
\begin{aligned}
\tau_{\max } & =\left(16 \times \mathrm{T}_{\mathrm{e}}\right) /\left(\pi \times \mathrm{d} \mathrm{d}^{3}\right) \\
& =\left(16 \times 96.62 \times 10^{3}\right) /\left(\pi \times 57^{3}\right) \\
& =2.6571 \mathrm{~N}-\mathrm{mm}^{2}
\end{aligned}
$$

As $T_{\text {max }}<T_{\text {per }}$, design is safe.

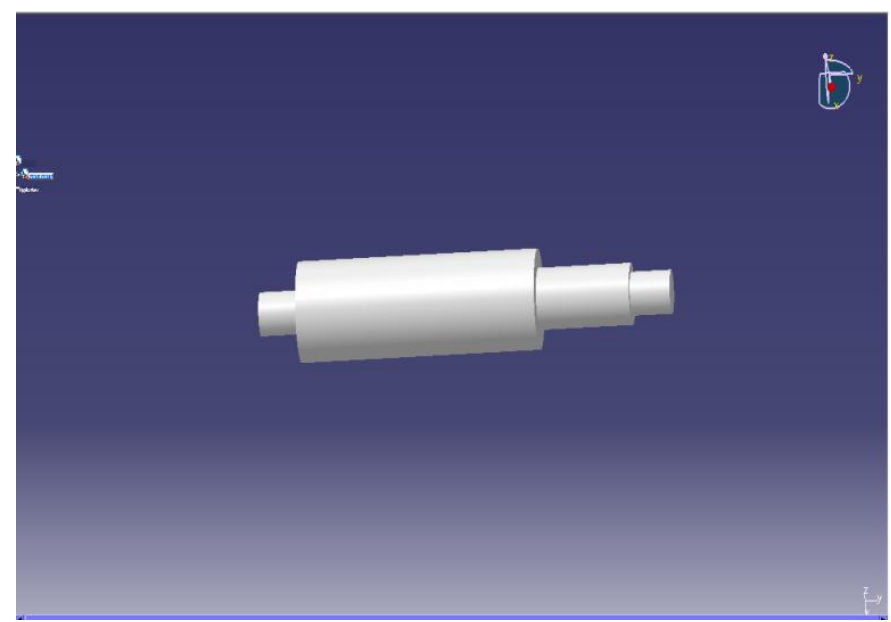

Fig 8

\subsection{Power Screw}

Material: EN9 C45

Dimensions: Nominal Diameter $(\mathrm{d})=10 \mathrm{~mm}$

Core Diameter $\left(\mathrm{d}_{\mathrm{c}}\right)=8 \mathrm{~mm}$

Mean Diameter $\left(\mathrm{d}_{\mathrm{m}}\right)=9 \mathrm{~mm}$

Pitch $=2 \mathrm{~mm}$

Length $=70 \mathrm{~mm}$

No. of Starts $=1$

$$
\text { Lead }=2 \mathrm{~mm}
$$

$$
1=N_{t} \times P
$$

\section{Design Procedure}

Torque Required to lower the load Against Friction:

$$
\mathrm{T}_{\mathrm{t}}=\frac{\mathrm{W} \times \mathrm{dm} \times \tan (\varnothing-\lambda)}{2}
$$

Now,

$$
\begin{aligned}
& \text { Lead Angle } \lambda \text { is, } \\
& \qquad \lambda=\tan ^{-1}\left[\left(\mathrm{px} \mathrm{N}_{\mathrm{t}}\right) /\left(\pi \mathrm{xd}_{\mathrm{m}}\right)\right]
\end{aligned}
$$

$$
\begin{aligned}
& \lambda=\tan ^{-1}[(2 \times 1) /(\pi \times 9)] \\
& \lambda=4.046^{0}
\end{aligned}
$$

Friction Angle :-

$$
\begin{aligned}
& \varnothing=\tan ^{-1}(\mu) \\
& =\tan ^{-1}(0.14) \\
& =7.9696^{\circ}
\end{aligned}
$$

$\mu=0.14$ V.B.Bhandari (p. n .194)

Torque is ,

$$
\begin{array}{r}
\mathrm{T}_{\mathrm{t}}=\frac{400 \times 9 \tan (7.96-4.04)}{2} \\
=123.34 \mathrm{~N}-\mathrm{mm}
\end{array}
$$

Torque required to overcome collar friction:-

) / 2

$$
\mathrm{T}_{\mathrm{t}}=\left(\mu_{\mathrm{c}} \times \mathrm{w} \times\left[\mathrm{R}_{0}+\mathrm{R}_{\mathrm{i}}\right]\right.
$$

$$
\begin{aligned}
& \mathrm{R}_{0}=\text { Outer Radius of Collar }=7.5 \mathrm{~mm} \\
& \mathrm{R}_{\mathrm{i}}=\text { Inner radius of collar }=5 \mathrm{~mm}
\end{aligned}
$$

$$
\begin{aligned}
\mathrm{T}_{\mathrm{t}} & =\frac{0.15 \times 400 \times[7.5+5]}{2} \\
& =375 \mathrm{~N}-\mathrm{mm}
\end{aligned}
$$

Total torque required,

$$
\begin{aligned}
\mathrm{T} & =\mathrm{T}_{\mathrm{t}}+\mathrm{T}_{\mathrm{c}} \\
& =123.34+375 \\
\mathrm{~T} & =498.34 \mathrm{~N}-\mathrm{mm}
\end{aligned}
$$

Stress in Screw Body ;

Direct Compressive or Tensile Stress $(\sigma)$

Torsional Shear Stress $(\tau)$

1. Direct Compressive or Tensile Stress,

$$
\begin{aligned}
\sigma= & \frac{\mathrm{w}}{\frac{\pi(\mathrm{dc}) 2}{4}} \\
& =\frac{400}{\frac{\pi}{4}\left(8^{3}\right)} \\
& =7.95 \mathrm{~N} / \mathrm{mm}^{2}
\end{aligned}
$$

2. Torsional Shear Stress,

$$
\begin{array}{r}
\tau=\frac{16 \mathrm{~T}}{\pi(\mathrm{dc}) 3} \\
=\frac{16 \times 498.34}{\pi \times 8^{3}}
\end{array}
$$

Now,

Permissible Compressive Stress $=110 \mathrm{~N} / \mathrm{mm}^{2}$ Permissible Shear Stress $=65 \mathrm{~N} / \mathrm{mm}^{2}$

As,

$$
\begin{aligned}
& \sigma_{\max }<\sigma_{\text {per }} \\
& \tau_{\max }<\tau_{\text {per }}
\end{aligned}
$$

Since, Design of Screw body is safe.

Stress in Screw Threads:-

Direct Shear stress in screw threads $\left(\tau_{\mathrm{s}}\right)$

Direct shear stress in nut threads $\left(\tau_{\mathrm{h}}\right)$

Bearing pressure

1. Direct Shear stress in screw threads

$$
\tau_{\mathrm{s}}=\frac{\mathrm{W}}{\pi \times \mathrm{d}_{\mathrm{c}} \times \mathrm{t} \times \mathrm{z}}
$$


$z=$ No. of threads in engagement

$\mathrm{Z}=\frac{\text { height of nut }}{\text { pitch of threads }}$

$=\mathrm{h} / \mathrm{p}$

$=20 / 2$

$=10$

$\tau_{\mathrm{s}}=\frac{400}{\pi \times 8 \times 1 \times 10}$

$\tau_{\mathrm{s}}=1.5915 \mathrm{~N} / \mathrm{mm}^{2}$

2. Direct shear stress in nut threads $\left(\tau_{\mathrm{h}}\right)$

$$
\begin{gathered}
\tau_{\mathrm{h}}=\frac{\mathrm{W}}{\pi \times \mathrm{d} \times \mathrm{t} \times \mathrm{z}} \\
\tau_{\mathrm{h}}=\frac{400}{\pi \times 10 \times 1 \times 10} \\
\tau_{\mathrm{h}}=1.2732
\end{gathered}
$$

3. Bearing pressure

$$
\begin{aligned}
P_{B} & =\frac{W}{\frac{\pi}{4}\left(d^{2}-d_{c}^{2}\right) \times 10} \\
P_{B} & =\frac{400}{\frac{\pi}{4}\left(10^{2}-8^{2}\right) \times 10} \\
P_{B} & =1.4147 \mathrm{~N} / \mathrm{mm}^{2}
\end{aligned}
$$

As ,

Unit bearing pressure $=1$ to $1.5 \mathrm{~N} / \mathrm{mm} 2$

(p.n.196)

$$
\left(\mathrm{P}_{\mathrm{b}}\right)_{\max }<\left(\mathrm{P}_{\mathrm{b}}\right)_{\text {per }}
$$

Our design is screw threads are safe.

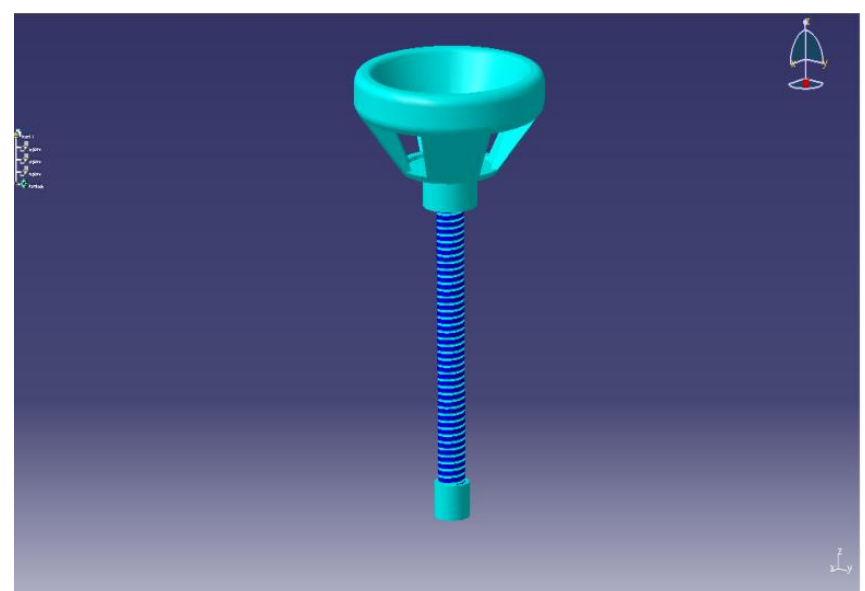

Fig 9

\subsection{Spur Gear Pair}

Material: EN-36 $\left(13 \mathrm{~N}_{1} 3 \mathrm{C}_{\mathrm{r}} 80\right)$

Design procedure:

$\mathrm{S}_{\text {up }}=\mathrm{S}_{\mathrm{ug}}=900$ to $1200 \mathrm{~N} / \mathrm{mm}^{2}$ \{ P.S.G.Design Data Book $\mathrm{BHN}=600$ to620

P.No. 8.4 \}

Since pinion and gear are made up of same material Therefore pinion is weaker

Now $\sigma_{\mathrm{bp}}=\mathrm{S}_{\mathrm{utp}} / 3$

$$
\begin{gathered}
\sigma_{\mathrm{bp}} \times \mathrm{Y}_{\mathrm{p}}=350 \times[0.484-(2.87 / 18)] \\
=113.59 \mathrm{~N} / \mathrm{mm}^{2}
\end{gathered}
$$

1. Beam Strength

$$
\begin{aligned}
& \mathrm{F}_{\mathrm{b}}=\sigma_{\mathrm{bp}} \times \mathrm{Y}_{\mathrm{p}} \times \mathrm{b} \times \mathrm{m} \\
& =350 \times 0.3245 \times 10 \mathrm{~m} \times \mathrm{m} \\
& =1135.94 \mathrm{~m}^{2} \mathrm{~N}
\end{aligned}
$$

2. Wear Strength

Ratio factor For External gear Pair,

$$
\begin{aligned}
& Q=\frac{2 z_{p}}{z_{p}+z_{g}} \\
= & \frac{2 \times 24}{24+18} \\
= & 1.142
\end{aligned}
$$

Load Stress Factor

$$
\begin{gathered}
\mathrm{K}=0.16[\mathrm{BHN} / 100]^{2} \\
\mathrm{~K}=0.16[610 / 100]^{2} \\
\mathrm{~K}=5.9536
\end{gathered}
$$

$$
\begin{aligned}
\therefore \mathrm{F}_{\mathrm{w}} & =\mathrm{d}_{\mathrm{p}} \times \mathrm{b} \times \mathrm{k} \times \mathrm{Q} \\
& =18 \mathrm{~m} \times 10 \mathrm{~m} \times 5.9563 \times 1.142 \\
& =1223.82 \mathrm{~m}^{2} \mathrm{~N}
\end{aligned}
$$

Since $\mathrm{F}_{\mathrm{b}}<\mathrm{F}_{\mathrm{w}}$

$\therefore$ Gear pair is weaker in Bending

2) Effective Load

$$
\begin{aligned}
\mathrm{V} & =\frac{\pi \times \mathrm{d}_{\mathrm{p}} \times \mathrm{n} \times \mathrm{p}}{60 \times 10^{3}} \\
& =1.3571 \mathrm{~m} \mathrm{~N} / \mathrm{mm}^{2} \\
\mathrm{~F}_{\mathrm{t}} & =\frac{\mathrm{p}}{\mathrm{v}} \\
& =\frac{746}{1.3571 \mathrm{~m}} \\
& =\frac{549.0}{\mathrm{~m}} \mathrm{~N} \\
\mathrm{k}_{\mathrm{v}} & =\frac{6}{6+\mathrm{v}} \quad \ldots \ldots .\{\mathrm{v}
\end{aligned}
$$
\{velocity Factor gears manufactured by hobbing,shaping or , milling

$$
\begin{aligned}
& \mathrm{k}_{\mathrm{v}}=\frac{6}{6+1.3571 \mathrm{~m}} \\
& \mathrm{~F}_{\text {eff }}=\mathrm{k}_{\mathrm{a}} \times \mathrm{k}_{\mathrm{b}} \times \mathrm{F}_{\mathrm{t}} \\
& \mathrm{k}_{\mathrm{a}}=\text { ApplicationFactor }=2.5
\end{aligned}
$$

. $\{$ V.B.Bhandari P.No. 675$\}$

$\mathrm{k}_{\mathrm{b}}=$ Load Distribution Factor $=1.3$

$$
\begin{aligned}
\mathrm{F}_{\text {eff }} & =\left[\frac{1.25 \times 1.3 \times 549.70}{\mathrm{~m}} \times \frac{(6+1.3571 \mathrm{~m})}{6}\right] \\
= & {\left[\frac{893(6+1.3571 \mathrm{~m})}{6 \mathrm{~m}}\right] } \\
= & {\left[\frac{5359.57+1212.24 \mathrm{~m}}{6 \mathrm{~m}}\right] }
\end{aligned}
$$

Estimation of module,

In order to avoid the bending failure,

$$
\mathrm{F}_{\mathrm{b}}=\mathrm{N}_{\mathrm{f}} \times \mathrm{F}_{\mathrm{eff}}
$$

$1135.94=\{1.75 \times[(5359.57+1212.24 \mathrm{~m}) / 6 \mathrm{~m}]\}$

$3894.65 \mathrm{~m}^{3}=5359.57+1212.24 \mathrm{~m}$

$$
\mathrm{m}=2.3 \approx 2.5
$$

Standard value of module under first choice is 2.5

Dimensions of gear pair

$$
\begin{aligned}
& \mathrm{m}=2.5 \\
& \mathrm{z}_{\mathrm{p}}=18 \\
& \mathrm{z}_{\mathrm{g}}=24 \\
& \mathrm{~b}=10 \mathrm{~m}=10 \times 2.5=25 \mathrm{~mm} \\
& \mathrm{~d}_{\mathrm{p}}=\mathrm{m} \times \mathrm{z}_{\mathrm{p}}=2.5 \times 18=45 \\
& \mathrm{~d}_{\mathrm{g}}=\mathrm{m} \times \mathrm{z}_{\mathrm{g}}=2.5 \times 24=60
\end{aligned}
$$




$$
\begin{aligned}
& \mathrm{a}=\frac{\mathrm{d}_{\mathrm{p}} \times \mathrm{d}_{\mathrm{g}}}{2} \\
& \mathrm{~h}_{\mathrm{a}}=1 \mathrm{~m}=2.5 \mathrm{~mm} \\
& \mathrm{~h}_{\mathrm{f}}=1.25 \mathrm{~m}=3.125 \mathrm{~mm}
\end{aligned}
$$

Precise estimation of dynamic load by Buckingham's equation

For grade 6,

$$
\begin{aligned}
& e=8+0.63(\mathrm{~m}+0.25(\sqrt{d}) \\
& e_{p}=8+0.63\left(\mathrm{~m}+0.25\left(\sqrt{d_{p}}\right)\right. \\
& e_{p}=10.6315 \mu \mathrm{m} \\
& e_{g}=8+0.63\left(\mathrm{~m}+0.25\left({ } d_{g}\right)\right. \\
& e_{g}=10.7949 \mu \mathrm{m} \\
& e=e_{p}+e_{g} \\
& e=21.4264 \times 10^{-3}
\end{aligned}
$$

Buckingham's Equation for the dynamic load in tangential direction,

$$
\begin{aligned}
& \mathrm{F}_{\mathrm{d}}=\left[21 \mathrm{v}\left(\mathrm{bC}+\mathrm{F}_{\mathrm{tmax}}\right)\right] /[21 \mathrm{v}+\sqrt[2]{\mathrm{bc}+\mathrm{Ftmax}}] \\
& \mathrm{F}_{\mathrm{t}}=549.70 / \mathrm{m} \\
&= 219.98 \mathrm{~N} \\
& \mathrm{~F}_{\mathrm{tmax}}=\mathrm{k}_{\mathrm{a}} \times \mathrm{k}_{\mathrm{m}} \times \mathrm{f}_{\mathrm{t}} \\
& \mathrm{F}_{\mathrm{tmax}}= 1.25 \times 1.3 \times 219.98 \\
& \mathrm{~F}_{\mathrm{tmax}}=357.46 \mathrm{~N} \\
& \mathrm{v}=1.3571 \times 2.5 \\
&=3.3927 \mathrm{~m} / \mathrm{s} \\
& \mathrm{c}=11500 \mathrm{e} \\
&=11500 \times 21.4246 \times 10^{-3} \\
&=246.376 \mathrm{~N} / \mathrm{mm}
\end{aligned}
$$$$
\left.\mathrm{F}_{\mathrm{d}}=[21 \times 3.3927(25 \times 246.376)+357.46)\right] /[21 \times 3.3927+
$$$$
\sqrt[2]{(25 \times 246.376)+357}]
$$$$
F_{d}=464304.76 / 151.9737
$$$$
\mathrm{Fd}=3055.1637 \mathrm{~N}
$$

$$
\begin{aligned}
& \text { Available Factor of Safety } \\
& \begin{aligned}
\mathrm{F}_{\mathrm{eff}}= & \mathrm{F}_{\mathrm{tmax}}+\mathrm{F}_{\mathrm{d}} \\
= & 357.46+3055.1637 \\
= & 3412.62 \mathrm{~N}
\end{aligned} \\
& \mathrm{~F}_{\mathrm{w}}=1223.82 \mathrm{~m}^{2} \\
& \mathrm{~F}_{\mathrm{b}}=1135.94 \mathrm{~m}^{2}
\end{aligned}
$$

Available factor of saftey,

$$
\begin{aligned}
\mathrm{N}_{\mathrm{f}} & =\frac{\mathrm{f}_{\mathrm{b}}}{\mathrm{f}_{\text {eff }}} \\
& =\frac{1135.94 \times(2.5)^{2}}{3412.62} \\
& =2.08 \\
\mathrm{~N}_{\mathrm{f}} & =2.08>1.75
\end{aligned}
$$

As the available factor of safety is higher than the required factor of safety

$\therefore$ Design is safe

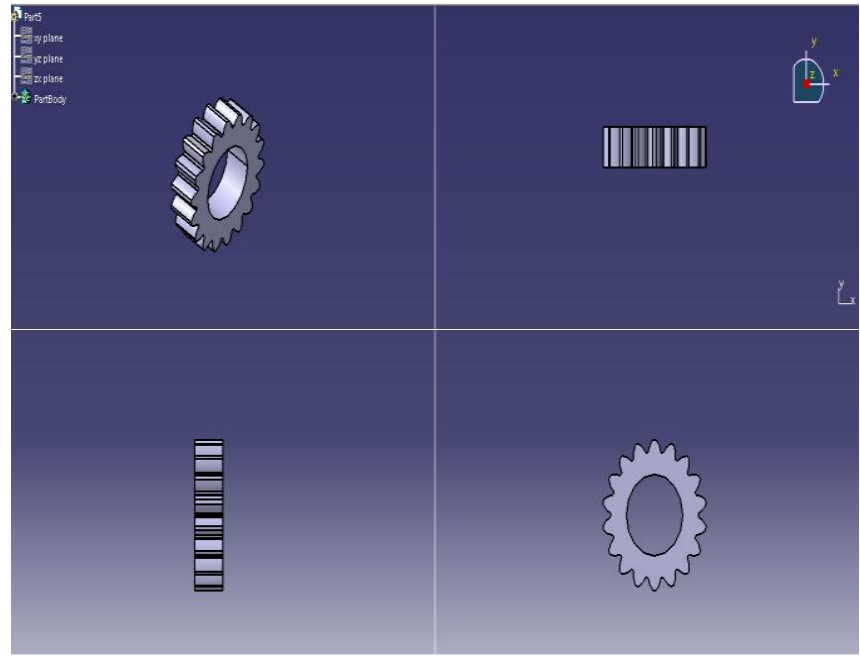

Fig 10

\subsection{Square Key}

Material: $48 \mathrm{C} 8$

Design procedure:

Allowable shear stress for key,

$$
\begin{aligned}
\tau_{\mathrm{sk}}= & 0.3 \times \mathrm{S}_{\mathrm{yt}} \\
= & 0.3 \times 350 \\
= & 105 \mathrm{~N} / \mathrm{mm}^{2}
\end{aligned}
$$

Let us take,

$$
\sigma_{\mathrm{c}}=2 \tau_{\mathrm{sk}}
$$

For square key,

1. Width of $\operatorname{key}(w)=d / 4$

$$
\begin{aligned}
& \begin{array}{c}
=20 / 4 \\
=5 \mathrm{~mm}
\end{array} \\
& \begin{array}{l}
=20 / 4 \\
=5 \mathrm{~mm}
\end{array} \\
& \begin{array}{l}
\text { 2. height of key }(\mathrm{h})=\mathrm{d} / 4 \\
\text { Considering crushing of key: } \\
\sigma_{\mathrm{c}}=4 \mathrm{t} /(\mathrm{d} \times \mathrm{h} \times 1) \\
210=\left(4 \times 89.047 \times 10^{3}\right) /(20 \times 5 \times 1) \\
1=16.96 \mathrm{~mm}
\end{array} \\
& \begin{array}{c}
\tau_{\mathrm{sk}}=\frac{2 T}{d \times w \times l} \\
105=\left(2 \times 89.047 \times 10^{3}\right) /(20 \times 5 \times 1) \\
1=16.96 \mathrm{~mm}
\end{array} \\
& \begin{array}{c}
1=1.5 \mathrm{~d}=1.5 \times 20 \\
=30 \mathrm{~mm}
\end{array}
\end{aligned}
$$

Select the larger value, $1=30 \mathrm{~mm}$.

\section{WORKING OF MACHINE}

1. 3 phase, 1 HP, D.C. motor is started with the 3 phase supply connection. The motor is having in built gearbox to achieve the required speed reductions.

2. The plate to be bend is placed in between the rollers and with the help of power screw necessary clamping force is applied on sliding block which is placed in the housing to provide proper placing of the plate in between the rollers. 
3. On the motor shaft, sprocket is mounted. With the help of chain and sprocket arrangement bottom rollers start to revolve on the starting the motor.

4. Spur gear pair is mounted on the roller shafts. Gear pair is utilized only for motion transmission from bottom rollers to top roller. Thus top roller also revolves.

5 . Hence now the plate is bent into the required shape after passing through the rollers.

6. Now the motor is switched off and clamping force is released to remove the final product from the machine.

7. A small undercut is provided on the top roller for easy removal of cylindrical shape component after manufacturing.

\section{STRESS ANALYSIS OF PLATE}

\subsection{Sress and Bending Moment Acting on the Plate}

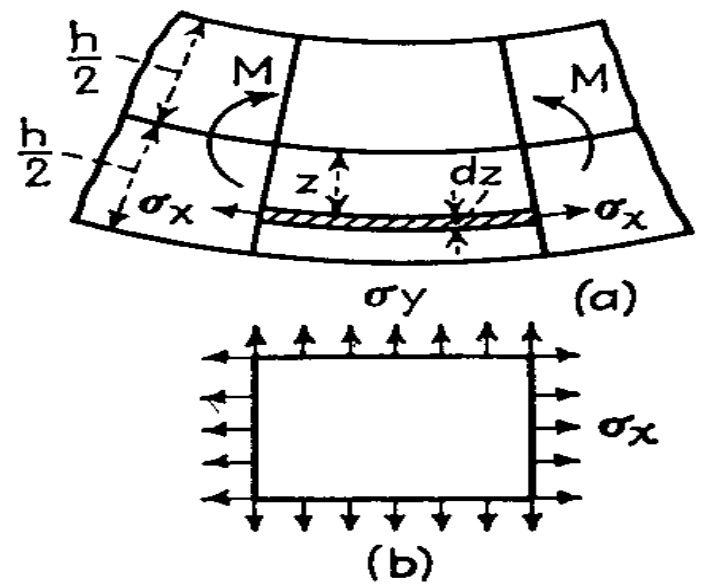

Fig. 11 Stress \& Moments

Where,

$\mathrm{h}=$ thickness of the plate.

$\underline{\sigma}_{\underline{x}}=$ bending stress

$\mathrm{M}=$ bending moment

$\mathrm{W}=$ deflection in $\mathrm{z}$ direction

$\mathrm{Z}=$ distance of the strip from neutral axis.

According to Hooke's law unit elongations $€_{\mathrm{x}}$ and $€_{\mathrm{y}}$ in terms $\sigma_{\mathrm{x}}$ and $\sigma_{\mathrm{y}}$ shown shaded in fig (a). are

$$
\begin{aligned}
& €_{\mathrm{x}}=\left(\sigma_{\mathrm{x}} / \mathrm{E}\right)-\left(\mu \sigma_{\mathrm{y}} / \mathrm{E}\right) \\
& €_{\mathrm{y}}=\left(\sigma_{\mathrm{y}} / \mathrm{E}\right)-\left(\mu \sigma_{\mathrm{x}} / \mathrm{E}\right)
\end{aligned}
$$

Where, $\mathrm{E}=$ modulus of elasticity

$$
\mu=\text { Poisson's ratio }
$$

lateral strain in y direction must be zero in order to maintain continuity in the plate during bending, from which it follows from eq (1) that $\sigma_{y}=\mu \sigma_{x}$. Substituting this value in the first of eq $(1), \quad €_{x}=\left[\left(1-\mu^{2}\right) \sigma_{x}\right] / E$

$$
\begin{aligned}
& \underline{\sigma}_{\underline{x}}=\left(€_{\mathrm{x}} \times \mathrm{E}\right) /\left(1-\mu^{2}\right) \\
& \sigma_{\mathrm{x}}=\left[\mathrm{E} /\left(1-\mu^{2}\right)\right]\left[-\mathrm{z} \times\left(\mathrm{d}^{2} \mathrm{w} / \mathrm{dx}^{2}\right)\right]
\end{aligned}
$$

Where, $\sigma_{x}=$ bending stress.

Bending moment in the elemental strip,

$\mathrm{M}=\int_{-h / 2}^{h / 2} \mathrm{z} \sigma \mathrm{xdz}$

$$
=\int_{-h / 2}^{h / 2}\left[\mathrm{E} /\left(1-\mu^{2}\right)\right]\left[-\mathrm{z}^{2} \mathrm{x}\left(\mathrm{d}^{2} \mathrm{w} / \mathrm{dx}^{2}\right)\right] \mathrm{dz}
$$

$$
\begin{gathered}
=\left[-\mathrm{Eh}^{3} / 12 \mathrm{x}\left(1-\mu^{2}\right)\right]\left[\mathrm{d}^{2} w / \mathrm{dx}^{2}\right] \\
\mathrm{M}=\mathrm{D} \times\left(\mathrm{d}^{2} w / \mathrm{dx}^{2}\right)
\end{gathered}
$$

Where,

$$
\begin{aligned}
\mathrm{D} & =\left[\mathrm{Eh}^{3} / 12 \times\left(1-\mu^{2}\right)\right] \\
& =\text { flexural rigidity of the plate. }
\end{aligned}
$$

\subsection{Sheet Metals}

1. Stainless steel: this is an alloy of steel with nickel, chromium and traces of other materials. It has good corrosion resistance and can be welded easily. This is used in dairies, food processing and kitchen wares etc.

2. Galvanized iron: zinc coated iron is known as galvanized iron. The zinc coating resist the rust, improves the appearance of the metal and permits it to be solder with greater ease. Articles such as pans, furnaces, heating ducts are mainly made from GI sheets.

3. Copper: copper sheets are available either as cold rolled or hot rolled sheets. Cold rolled sheets being worked easily are commonly used in sheet metal shops and are resistant to corrosion. Roof flashings and hoods are some of the common example of copper sheets.

4. Aluminium: It cannot be used in pure form but it is used with a very small amount of copper, silicon, manganese and iron. it is now widely used in the manufacture of number of articles such as household appliances, lighting fixtures ,filling used in doors, windows and on many electrical and transport industries.

\subsection{Spring Back}

1. It is generally caused due to variations of bending stress. Spring back reduces towards the center of the sheet and becomes zero at its neutral axis.

2. The metal is stressed to a value below its elastic limits. The metal creates a narrow elastic bend on both the sides of neutral axis. The metal deviates further away from the neutral axis stressed beyond its yield point and plastically deformed, but it slightly regains its present form

3 . Thus, the force applied on the top roller is calculated considering the spring back action. So we have to apply more force than the required force in order to avoid spring back

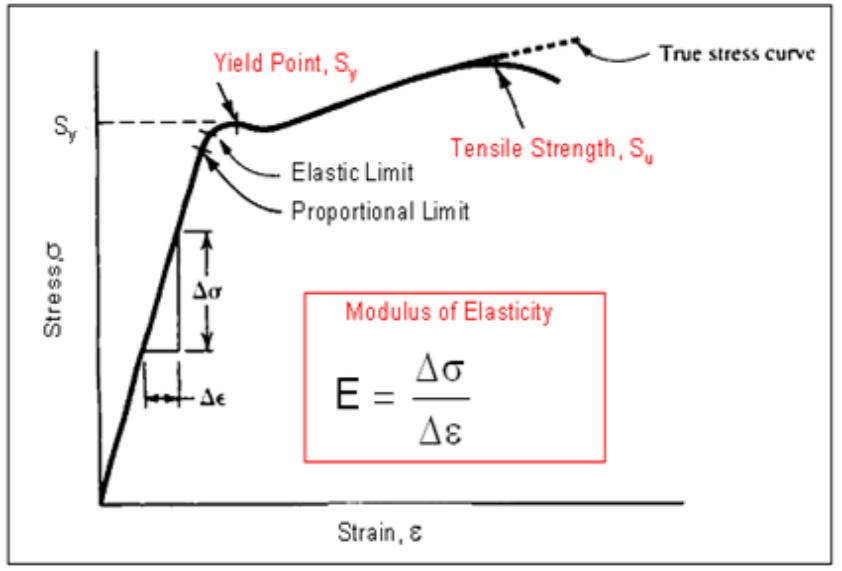

Fig 12 Stress-Strain Curve for Steel 


\section{SERVICE LIFE OF VARIOUS COMPONENTS}

As PB bush and rollers are subjected to continuos loading and frictional forces.hene more wear and tear takes place at both of these components. Thus life of above components is found out as below:

\subsection{P.B.BUSH}

Table 5 : PV Chart

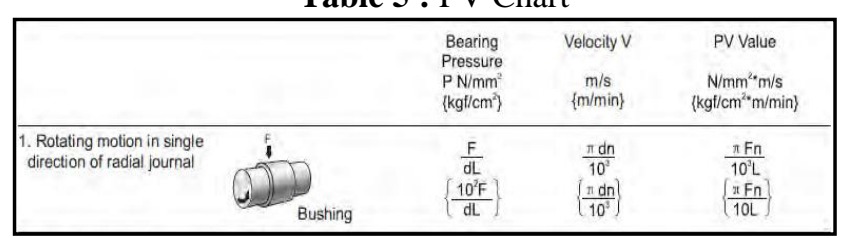

Calculation:-

$$
\mathrm{PV}=\frac{\pi \mathrm{Fn}}{10^{3} \mathrm{~L}}
$$

Where,

$$
\begin{aligned}
& \mathrm{F}=\operatorname{vertical} \operatorname{load}(\mathrm{N}) \\
& \mathrm{n}=\text { no. of rotation } \\
& \mathrm{L}=\text { bush length }
\end{aligned}
$$

$\mathrm{PV}=\frac{\pi \times 449.05 \times 80}{10^{3} \times 20}$

$\mathrm{PV}=5.643<10$

Where, maximum PV value for the PB bush should be 10 .

It sustain for 5 years approximately.

\subsection{Rubber}

Bottom rollers are coated with synthetic rubber to increase its working life and to provide proper grip for the plate to roll between the rollers. Hardness of the rubber used is 7585 shore-A. Hence it can sustain for 5-7 years approximately.

\section{TROBLE SHOOTING, INVOATIONS}

\begin{tabular}{|c|c|c|c|}
\hline & shape & & \\
\hline 4. & $\begin{array}{l}\text { The rolls } \\
\text { revolves in } \\
\text { the housing }\end{array}$ & $\begin{array}{c}\text { Lack of } \\
\text { proper } \\
\text { lubrication }\end{array}$ & $\begin{array}{l}\text { It should be } \\
\text { properly } \\
\text { lubrication }\end{array}$ \\
\hline 5. & $\begin{array}{c}\text { Inter locking } \\
\text { or Jamming } \\
\text { of gears }\end{array}$ & $\begin{array}{l}\text { Due to lack } \\
\text { of proper } \\
\text { alignment \& } \\
\text { lubrication }\end{array}$ & $\begin{array}{l}\text { While assembling } \\
\text { the alignment of } \\
\text { the rolls should be } \\
\text { checked \& the } \\
\text { gears should be } \\
\text { properly lubricated }\end{array}$ \\
\hline 6. & $\begin{array}{c}\text { Lubrication } \\
\text { of the } \mathrm{M} / \mathrm{C} \\
\text { while } \\
\text { bending }\end{array}$ & $\begin{array}{l}\text { The base } \\
\text { frame is not } \\
\text { properly } \\
\text { bolted to this } \\
\text { sides }\end{array}$ & $\begin{array}{c}\text { All the bolts are } \\
\text { properly tightened } \\
\& \text { checked before } \\
\text { the operations } \\
\text { starts }\end{array}$ \\
\hline
\end{tabular}

\subsection{Troble Shooting}

Table 6 Troble Shooting

\begin{tabular}{|c|c|c|c|}
\hline Sr.No & Faults & Causes & $\begin{array}{c}\text { Suggested } \\
\text { Remedies }\end{array}$ \\
\hline 1. & $\begin{array}{c}\text { Oval Shape } \\
\text { Of the plate } \\
\text { after rolling }\end{array}$ & $\begin{array}{c}\text { Pinching is } \\
\text { not done }\end{array}$ & $\begin{array}{c}\text { Pinching operation } \\
\text { should done with } \\
\text { the help of suitable } \\
\text { gauge }\end{array}$ \\
\hline 2. & $\begin{array}{c}\text { Waves are } \\
\text { formed on } \\
\text { the surface at } \\
\text { plates }\end{array}$ & $\begin{array}{c}\text { Too much of } \\
\text { pressure is } \\
\text { applied on } \\
\text { the plates }\end{array}$ & $\begin{array}{c}\text { The pressure } \\
\text { should be given } \\
\text { gradually }\end{array}$ \\
\hline 3. & $\begin{array}{c}\text { Bending is } \\
\text { not proper } \\
\text { i.e. after } \\
\text { bending the } \\
\text { plates comes } \\
\text { back to its } \\
\text { original }\end{array}$ & $\begin{array}{c}\text { This is due to } \\
\text { spring back. }\end{array}$ & $\begin{array}{c}\text { We have to over } \\
\text { bend it. For this } \\
\text { more force than } \\
\text { monply }\end{array}$ \\
\hline \multicolumn{2}{|c|}{ the required force } \\
\hline
\end{tabular}

\subsection{Innovations}

1. Rubberizing : Rubberizing is done to the bottom rollers for reducing the friction, slipage between the rollers and plate and for maximizing the life of rollers.

2. Undercut : Undercut is given to the top roller for easy removal of manufactured part.

3. Bush : Bushes are used instead of bearings, as it distributes the load equally, When it comes in contact with steel machine shafts, it will not bind to steel because it is a dissimilar metal

4. Motor : Instead of using large no of gears we have utilized a single motor with gearbox to get the required speed reduction.

\section{ADVANTAGES AND APPLICATIONS}

\subsection{Advantages}

1. Operation of this machine is very simple.

2. Unit is compact so less space is required.

3. The diameter can be easily operate this machine.

4. The total cost of the machine is less.

5. Maintenance of this machine is very easy.

6. Easy to handle.

7. Time consumption is less.

8. Less effort \& productive.

9. Easy to install at any were.

10. Skilled workers are not required.

11. Convenient for mass production.

12. Less in weight.

\subsection{Applications}

Sheet Metal rolling machine is used to make:

1. Outer cylindrical casing for motors employed in submersible pumps.

2. Bodies of shells and condensers.

3. Dairy equipment.

4. It is also used to make cylinders of different diameters.

5. It is also used in bicycles. 


\section{CONCLUSION}

Design analysis of three roll pyramid type plate bending machine has been made considering a steel plate of width 80 $\mathrm{mm}$ and thickness $1 \mathrm{~mm}$.

Layout of the machine and the configuration is decided based on this analysis.

Forces on various parts/components are estimated.

Using the standard design procedure, various components of the machine is designed and the design details are shown in respective sections/chapters.

Thus we have manufactured a pyramid type plate bending machine.

\section{REFERENCES}

[1] Bendability Analysis for Bending of Steel Plates on 3-Roller Bending Machine, International Journal of Aerospace and Mechanical Engineering 2007, presented by Himanshu V. Gajjar, Anish H. Gandhi, Tanvir A Jafri and Harit K. Raval.

[2] Modeling and computation of the three-roller bending process of steel sheets, Journal of Mechanical Science and Technology 26 (1) (2012) 123 128, presented by Ahmed Ktari, Zied Antar, Nader Haddar and Khaled Elleuch. (Manuscript Received July 9, 2010; Revised December 13, 2010; Accepted September 18, 2011).

[3] Analytical Model For Prediction Of Force During 3Roller Multi-pass Conical Bending and its Experimental Verification, international journal of mechanical engineering and robotics research, ISSN 2278-0149S, VOL.1, NO.3, October 2012, presented by M K Chudasamal and H K Raval.

[4] Shigley J, "Mechanical Engineering Design", p44, International Edition, pub McGraw Hill, 1986, ISBN 0-07-100292-8.

[5] Gere, J. M. and Timoshenko, S.P., 1997, Mechanics of Materials, PWS Publishing Company. 